\title{
A Theoretical Background of loT Platforms based on FPGAs
}

\author{
Noor Kareem Jumaa \\ Computer Technology Engineering \\ Dept. \\ Al Mansour University College, Iraq
}

\author{
Omar Anwer Abdulhameed \\ Computer Technology Engineering \\ Dept. \\ Al Mansour University College, Iraq
}

\author{
Rasha Hassan Abbas \\ Computer Technology Engineering \\ Dept.
}

Al Mansour University College, Iraq

\begin{abstract}
IOT is a network of connected things through internet using IOT platform. Many of IOT systems have been build using microcontroller, IOT can be rebuild using more precise concept for embedded systems using FPGA. The working principles of FPGA are systolic array and pipelining that speed up data processing through parallelism. Using FPGA as IoT platform performed through connecting FPGA to the internet which achieved by companies that are manufacturing FPGA, and that covered by many researches and studies. This research will cover the main principles of how to build IoT platform using FPGA.
\end{abstract}

\section{General Terms}

FPGA, IoT, Internet.

\section{Keywords}

FPGAs vs CPUs, IoT Platforms, FPGA Gateway, Intel, Altera.

\section{INTRODUCTION}

Everything is in the way to be computerized and in present days, a lot of the objects are to be come smart objects. These smart objects are allowed to be on the network using platforms of the Recent Internet of Thing (IoT). "IoT is a smart information society that consists of smart devices; these devices can communicate with each other without human's intervention". IoT stands for a system that enables the devices to communicate with each other directly without intervention of humans [1] (Figure 1 illustrate the components of IoT system).

As shown in Figure 1, IoT systems consist of the following components:

- Sensors: a sensor could be a device, module, or subsystem. Its purpose is to discover the changes and the events in the environment and send the information signal to processor.

- Data: perform the information obtained from the sensors which is transferred to the cloud for processing, analyzing, and monitoring.

- Systems: IoT systems data are processed, analyzed, and monitored through the concept of systems whose perform the processing cloud programs for example Thingspeak IoT platform would analyze data of an IoT system through cloud Matlab Program.

- Internet connection: it is the internet connectivity where the microprocessor of an IoT system should be able to access the internet through either be connected directly to a modem that communicates with an Internet Service Provider (ISP) or the modem's internet connection would be shared via a Local Area Network (LAN).

- Platforms: a platform is a communication technology which available to enable the objects to be connected over networks like Bluetooth, Wi-Fi, Zigbee, 4G, and LTE.

- Things: are smart objects and devices whose could be connected together through the internet.

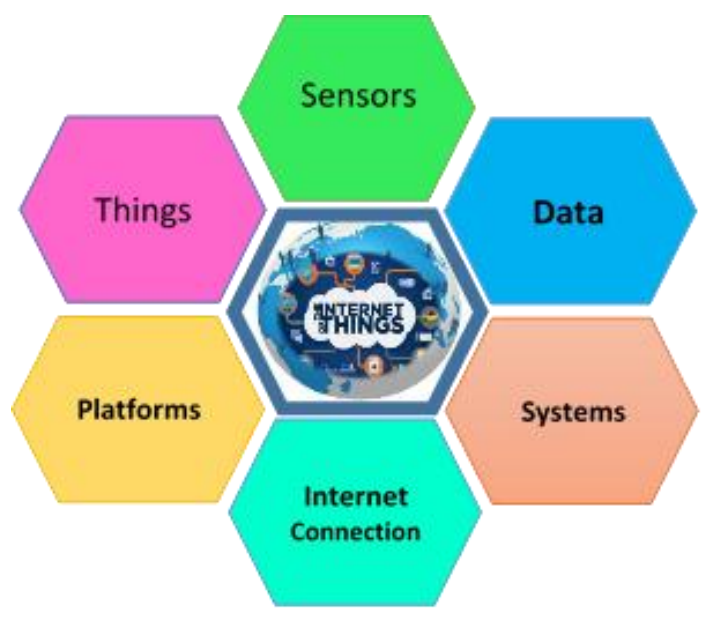

Figure 1: Components of IoT Systems

Things can be empowered to access the Internet as objects by using IoT. These objects are allowed to be on the network through the using of IoT platforms. There exist several platforms that provide different services such as notification, data analytic, business analytic, and many other services. Each platform offers a specific service $[1,2,3]$.

The term IoT was first coined by British technology pioneer Kevin Ashton in 1999 to demonstrate a system in which world devices could be connected to the Internet via sensors; he establishes a laboratory in MIT for connecting the objects through RFID. ITU is referred to IoT in the year 2005. In 2010 an IoT car was developed by Google; the car was fully connected to the Internet which could access the map and hence get by self-automated which performs an important project in IoT field. Bluetooth low energy protocol was developed in 2010, which enables objects to be connected with low consumption of power. In 2020 billions of devices are expected to be connected wirelessly as predicted by Cisco $[1,2,4]$.

In IoT systems, data should be transferred among the connected objects and the cloud. Many connectivity and communication technologies are available to enable the 
objects to be connected over networks like Bluetooth, Wi-Fi, Zigbee, 4G, and LTE. These technologies differ from other in speed, transmission, and power consumption. Based on the type of the application the technology that is used to provide connectivity and the constraints of the power, the chosen technology may vary. Table 1 lists the communication technologies of IoT platforms [2].

Table 1: List of Connectivity Technologies

\begin{tabular}{|l|l|l|}
\hline Technologies & Range / meters & Power \\
\hline Wi-Fi & $50-100$ & High \\
\hline Bluetooth & 10 & Low \\
\hline Zigbee & $10-100$ & Very low \\
\hline
\end{tabular}

As mentioned before, in IoT systems, the environment is monitored by sensing it while the data is transferred to the cloud by internet connectivity of the things. Thus, to achieve the fullest platforms of IoT, operating environment has to be developed to easy the process of the IoT system deployment [2].

Web services of FPGA based hardware have already been defined and realized. The embedded nature of FPGAs allows the developers to simply adjust those web services to actively interconnect with their surroundings for example, to achieve data measurement of real world or control different actuators. Such entities can have called environment aware Web services in difference to classical Web services that work on remote virtual or physical machines. In the present days, IP address is added to the VHDL targeted code design so as to make Internet of things (IOTs) enable VLSI Design as show in Figure $2[3,5,6,7]$.

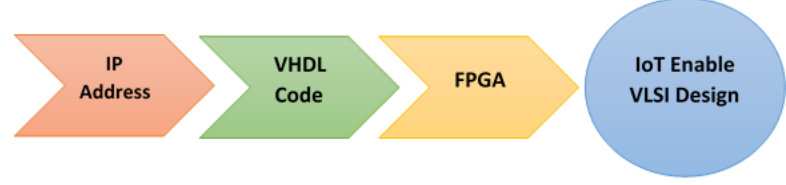

Figure 2: IoT Enable VLSI Design

This paper reviews a theoretical background of IoT platforms Design using FPGAs. The followed subsections of this paper is organized as follow: section II presents a literature review, section III discussing why to use FPGA for IoT platforms, section IV illustrates and discusses FPGAs vs CPUs, section $\mathrm{V}$ presents IoT platforms using FPGA, and section VI concludes the paper.

\section{LITERATURE REVIEW}

In [1], Internet of thing using FPGA had been surveyed and many structures of IoT system based on FPGA had been illustrated just like: motor control system, medical health care modeling system, cloud based communication module, and control plane interface based FPGA.

In [3], a review of technology paradigm for IoT on FPGA had been discussed. “

In this paper the application of IOT is examined in the FPGA platform, production deices Mars. It includes data collection, congeal, automation algorithms along with the implementation of the TCP / IP stack in FPGA with an interface cable or Wi-Fi Ethernet".
In [5], a review of FPGA implementation of internet of things has been discussed. "The main aim of this research is to highlight how the users can access the FPGA based design resources from anywhere. Thus we present a concept that shortens the application of momentarily unused resources for executing various tasks automatically".

In [8], Intel has developing solutions for the Internet of thing; in this white paper, Intel discuss building blocks for things which are: processors and chipsets, operating systems, data and things security, and network connectivity. Also Intel solutions for gateway has been discussed.

In [9], International lectrotechnical Commission (IEC) present white paper entitled IoT 2020: Smart and secure IoT platform which has been prepared by the IoT 2020 project team in the IEC Market Strategy Board (MSB). This paper discusses the architecture patterns and many other IoT features.

\section{WHY FPGA?}

Field Programmable Gate Array (FPGA) relatively provides short design cycle, high gate density, and low cost in electronic and electrical circuit design. FPGA "is a silicon chip containing an array of reprogrammable logic blocks (CLBs), which are connected together through a matrix of vast interconnection to form complex digital circuits". Designs of FPGA based systems are programmed in either Verilog or VHDL ((Very high speed integrated circuit) Hardware Description Language) in the process of being compiled into the design [10]

By Comparison to other methods of hardware building, FPGAs have two huge things going for them: First, they enable the designer to build exactly the hardware he/she needs, and the second is instead of having to use the same application specific standard product (ASSP) all the competitors are using, or having to undertake the cost, risk, and time of an application-specific integrated circuit (ASIC) design; But just as important, that ability to customize the FPGA means that often, in an FPGA, operations can performed in a softer, faster, more energy efficient manner than they could be done in the microprocessor cores of an ASSP [11].

Recent FPGAs comprise mixes of configurable "static random access memory" (SRAM), logic blocks, high-speed input/output pins, and routing. More precisely, an FPGA contains programmable logic elements called logic elements (LEs), along with a hierarchy of reconfigurable interconnects that allow the LEs to be physically connected to each other. The designer can configure LEs to do complex functions or simply perform basic logic gates, such as OR and AND [11].

\section{FPGAs vs CPUs}

Field Programmable Gate Array (FPGA) is selected as a Very Large Scale Integration (VLSI) platform device ever since, FPGA can provides certain computations at very high frequencies with systolic (pipelining) computing [12].

In computing, FPGAs can used as dedicated computers to achieve certain computations at very high frequencies. In nowadays, considerably, FPGAs have been improved the density, speed, and functionality, which makes them ideal for System On a Programmable Chip (SOPC) designs for a wide range of applications. Instead of costly multicore "Digital Signal Processing" (DSP) systems, designers can support from high density and high performance FPGAs [12, 13]. 
Generally, FPGAs are more flexible and cost effective than ASICs due to the following reasons:

\section{Costs and flexibility:}

Using of FPGAs, designers can implement any logical function that an ASIC can do but with the distinct advantage of updating the functionality after chip manufacture, which is desirable for many applications. FPGAs are more cost effective than ASICs because the customer can program FPGAs according to its requirements instead of contracting a vendor to design and construct an ASIC to meet its needs [11].

\section{Design time risk reduction versus speed:}

If the most advanced semiconductor process in the world have been set out to use, no matter the cost, it always could to design an ASIC that would run faster than the fastest available FPGA. But almost no one uses the most advanced process: Doing so would be very difficult, risky, and witheringly expensive. In fact, only a handful of ASSP companies leap on a new process as soon as it's available. Everyone else uses a process that is one, two, or three generations old. And the fact is, the fastest FPGA can compete directly with those older ASIC processes. And the FPGA brings reduced design work and far less risk [11].

\section{Parallel operation and order reduction:}

Modern designers have become relatively good at making tools that are smart enough to ease more complicated operations into simpler ones (this is known as order reduction) also, they have the ability to perform a complex operation in a series of instructions that operate simultaneously, which is known as parallel operation.

A microprocessor can do just about anything simply by executing its instructions. If the program says to multiply, the microprocessor loads the instruction from memory, decodes it, loads each number, multiplies them, and stores the result. Each one of those steps takes time and energy. But what if all you wanted to do was multiply a number by 2 ? That is just a shift operation. In general, if the operation was a multiplication operation and the designer know one of the coefficients is a constant value, he/she can reduce this complex operation to a simpler one, saving his/her processing time and power. Unlike FPGAs, Microcontrollers don't have the smarts to reduce multiplication to addition when possible, thus they have to perform the multiplication operation, resulting in slower execution speed and more power use [11].

FPGAs are unlike microprocessors; FPGAs are using hardware which is dedicated for logic processing and do not have an operating system. Different operations do not have to compete for the same processing resources since the processing paths are parallel $[1,14]$.

As a summary, FPGAs are better than CPUs due to the following reasons:

1. "Microprocessors are more complex than FPGAs"

2. "Microprocessors have fixed instructions while FPGAs don't"

3. FPGAs speed can be very fast, and multiple control loops can run on a single FPGA device at different rates.

Alike to its desktop CPUs that have on the die integrated GPU, Intel is taking a two-pronged tactic with its FPGA strategy: first, offering both hybrid CPU-FPGA processors and second, discrete Arria or Stratix brand FPGA devices on a PCI Express card. The hybrid CPU-FPGA device based on a Skylake generation CPU and Arria 10 FPGA (shown in Figure 3) [15].
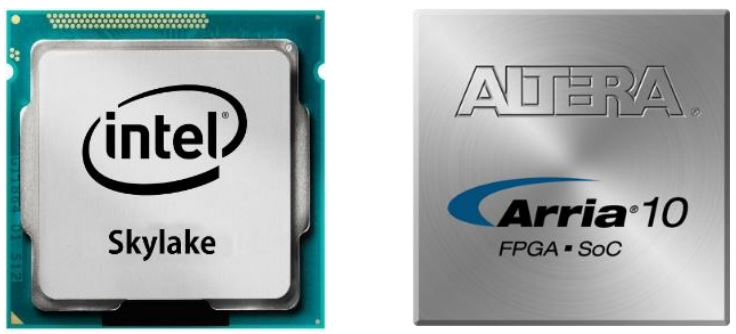

Figure 3: Skylake CPU and Arria 10 FPGA from Intel

"Intel Hybrid chips that combine FPGAs with their wellknown CPUs, achieving a goal set in 2010. Since the acquisition, Intel has used its new FPGA branch to focus on developing solutions for machine learning, encryption, big data processing, cloud computing, and accelerated computing with the reprogrammable hardware. Also available is a software stack to make working with the hardware much easier, whether it is being used for application development or acceleration development" [16].

Intel Stratix 10 (shown in Figure 4) is a hybrid CPU-FPGA chip which has the following features:

- $\quad 5.5 \mathrm{M}$ Logic Elements

- Heterogeneous 3D SiP Integration

- Intel 14 nm Tri-Gate

- Quad-Core Cortex-A53 ARM processor

- $2 X$ core performance

- $\quad$ Up to $70 \%$ lower power

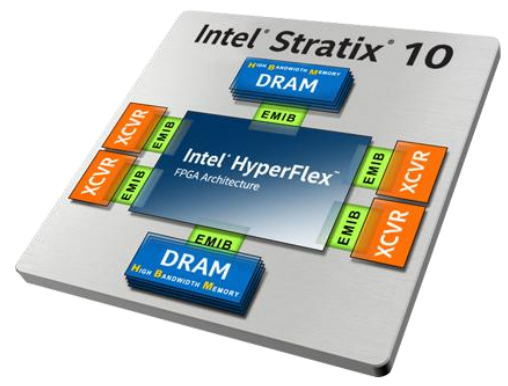

Figure 4: Intel Stratix 10 chip

\section{IOT PLATFORMS USING FPGA}

Intel FPGA (formerly Altera) offers many FPGAs based IoT systems. Building automation and smart city infrastructure, healthcare system infrastructure, smart grid control, car automotive systems, and machine vision systems are IoT systems based on FPGA and they can be seen at Altera web site $[1,17]$.

Altera offered many advantages to the manufactures, which are: $[1,17]$

1. Cost reduction: achieved by avoiding ASICs' extensive.

2. Time to market advantage: achieved by avoiding the risky and lengthy development cycle. 
3. Cost reduction and differentiation: achieved by integrating multiple ASSP functions into FPGAs.

4. Programmability: achieved during the design process and after equipment is in the field.
5. Reusability: achieved by using one hardware platform for various systems with one basic design.

6. Adaptability: achieved to multiple industry standards and protocols.

Figure 5 shows the components of FPGA based IoT platform.

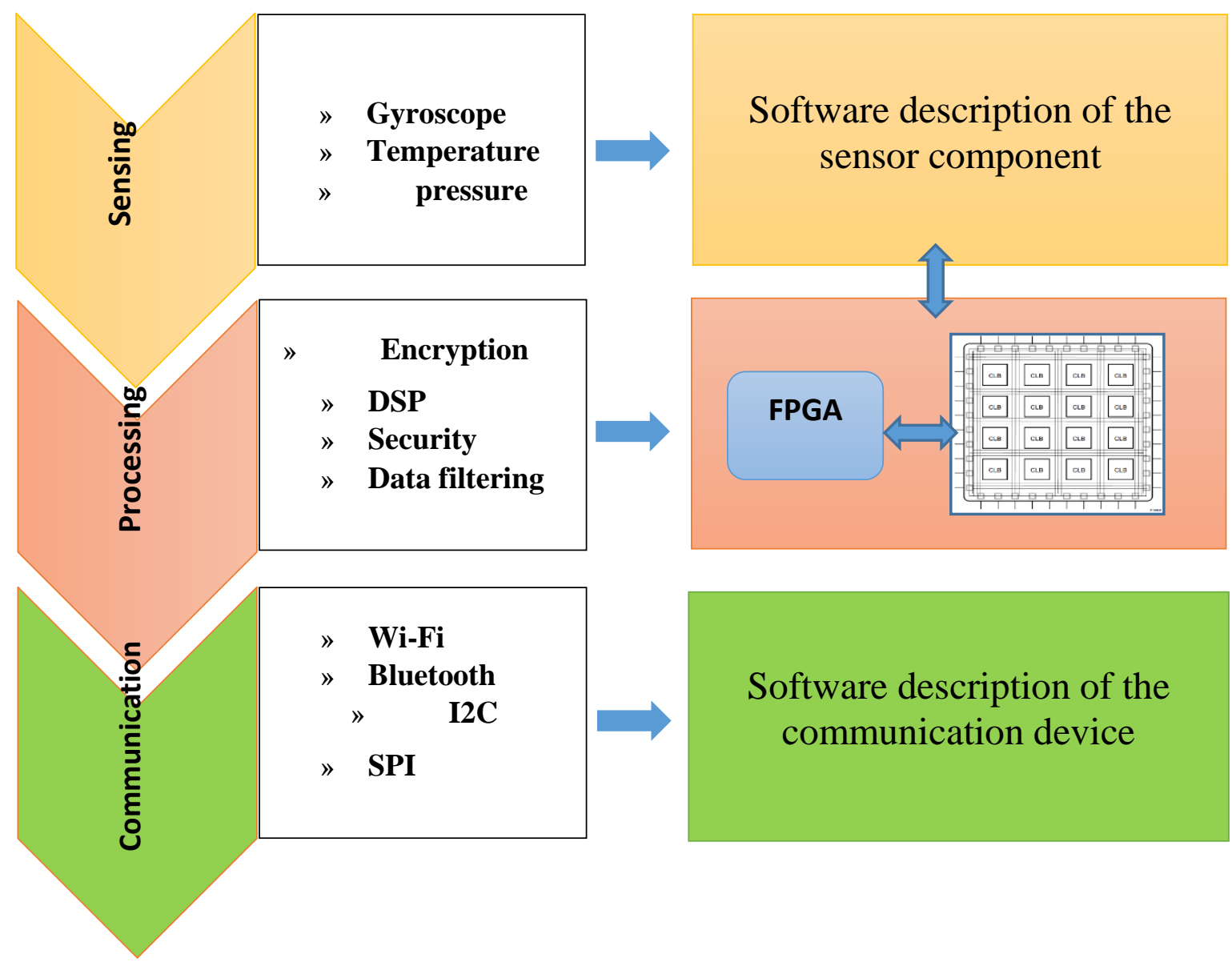

Figure 5: Components of FPGA based IoT platform

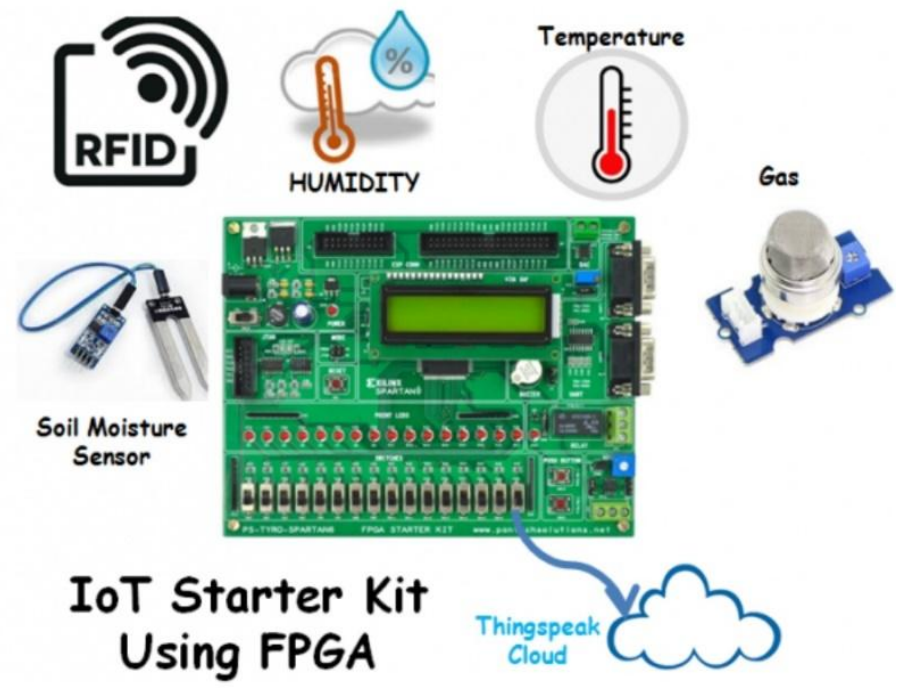

Figure 6: Illustration of Thingspeak Based FPGA Systems 
Fig. (6) shown above, an illustration of Thingspeak and FPGA connection is shown. Where Thingspeak is an IoT platform.

\section{CONCLUSIONS}

Pipelining and parallel processing are two sides of the same coin, so FPGA's access the operations very fast according to parallel processing concepts since their concepts of data processing is the pipelining.

This article is working on accommodating to use the FPGAs kit as an IoT platform. The embedded nature of FPGAs permits the designers to simply connect their surroundings things actively to web services. By comparing the usage of microcontroller and FPGA with IoT concepts, FPGA is more adaptability, reusability, and programmability. FPGAs are speeder than microcontrollers.

\section{REFERENCES}

[1] Noor Kareem Jumaa, "Survey: Internet of Thing Using FPGA", Iraq J. Electrical and Electronic Engineering, Vol.13, No.1, 2017.

[2] M. Sruthi and B. R. Kavitha, "A SURVEY ON IOT PLATFORM", International Journal of Scientific Research and Modern Education (IJSRME), ISSN(online): 2455-5630, Vol. I, Issue I, 2016.

[3] Ajay Rupani, Deepa Saini, Gajendra Sujediya, and Pawan Whig, "A Review of Technology Paradigm for IOT on FPGA", International Journal of Advanced Research in Computer and Communication Engineering, Vol. 5, Issue 9, 2016.

[4] RUPA CHANDRA, ASHA RATHEE, POOJA VERMA, and 4ARCHANA CHOUGULE, "GSM BASED HEALTH MONITORING SYSTEM", Proceedings of IRF International Conference, 13th, Pune, India, ISBN: 978-93-84209-04-9, 2014.

[5] Ajay Rupani and Gajendra Sujediya, "A Review of FPGA implementation of Internet of Things", International Journal of Innovative Research in Computer and Communication Engineering, Vol. 4, Issue 9, 2016.

[6] A. Ruta, R. Brzoza-Woch, and K. Zieli 'nski, "On fast developmentof FPGA-based SOA services-machine vision case study", DesignAutomation for Embedded Systems, vol. 16, no. 1, pp. 45-69, 2012.

[7] Robert Brzoza-Woch, PiotrNawrocki, "Reconfigurable FPGA-based embedded Web services as distributed computational nodes", Position Papers of the FEDCSIS. ŁO' DZ', Vol. 6, pp-159-164, 2015.

[8] Internet of Things Development: Intel White Paper, https://www.intel.com/content/www/us/en/internet-ofthings/white-papers/developing-solutions-for-iot.html.

[9] IoT 2020: Smart and secure IoT platform: International lectrotechnical Commission (IEC) White Paper, http://www.iec.ch/whitepaper/iotplatform/?ref=extfooter

[10] Noor Kareem Jumaa and Dr. Firas Ali Sabir, "Performance Analysis of FPGA - based LFSR-Pseudo Random Number Generator", Second Feministic Conference for Women and Science in Iraq, University of Baghdad, 2013.

[11] Andrew Moore and Ron Wilson, "FPGA for Dummies", 2nd Intel Special Edition, John Wiley \& Sons, 2017.

[12] Noor Kareem Jumaa, "FPGA Implementation of Programmable Systolic Array for Sinusoidal Sequence Generation", Communications on Applied Electronics (CAE), Foundation of Computer Science FCS, ISSN: 2394-4714, Volume 5, No.1, 2016.

[13] Rakesh Birle and Lalit Bandil, "Design and FPGA Implementation of Systolic Array Architecture for Matrix Multiplication", International Journal of Engineering and Advanced Technology (IJEAT), Vol. 1, ISSN: 2249-8958, 2012.

[14] Renee Robbins, "Advantages of FPGAs", http://www.controleng.com

[15] Network World, "Intel plans hybrid CPU-FPGA chips", 2017 , https://www.networkworld.com/article/3230929/datacenter/intel-unveils-hybrid-cpu-fpga-plans.html

[16] All about Circuits, "ntel to Introduce New CPU-FPGA Hybrid Chip Supported by Acceleration Stack", 2017, https://www.allaboutcircuits.com/news/intel-tointroduce-new-cpu-fpga-hybrid-chip-supported-byacceleration-stack/

[17] www.Altera.com.

[18] Kam Chuen Mak and Andrew Canis, "SoftwareProgrammable FPGA IoTPlatform”, Lattice Semiconductor, 2016. 